\title{
Dynamics and Expression of Solution of a Sixth Order Difference Equation
}

\author{
Abdul Khaliq ${ }^{1}$ \\ ${ }^{1}$ Department of Mathematics, Riphah Institute of Computing and Applied Sciences, Riphah International University, Lahore Campus, \\ Pakistan
}

\author{
Article Info \\ Keywords: Difference equation, Period- \\ icity, Global stability, Boundedness \\ 2010 AMS: 11D68, 12FO5, 14H40, \\ $14 H 50$ \\ Received: 29 April 2021 \\ Accepted: 10 September 2021 \\ Available online: 10 September 2021
}

\begin{abstract}
This paper deals with the solution behavior and periodic nature of the solutions of the difference equation

$$
s_{n+1}=\alpha s_{n}+\frac{\beta s_{n} s_{n-4}}{\gamma s_{n-4}+\delta s_{n-5}}, \quad n=0,1, \ldots
$$

where the initial conditions $s_{-5}, s_{-4}, s_{-3}, s_{-2}, s_{-1}, s_{0}$ are arbitrary positive real numbers and $\alpha, \beta, \gamma, \delta$ are positive constants. Also we obtain the closed form of the solutions of some special cases of this equation.
\end{abstract}

\section{Introduction}

This paper deals with the solution behaviour of the difference equation

$$
s_{n+1}=\alpha s_{n}+\frac{\beta s_{n} s_{n-4}}{\gamma s_{n-4}+\delta s_{n-5}}, \quad n=0,1, . .
$$

where the initial conditions $s_{-5}, s_{-4}, s_{-3}, s_{-2}, s_{-1}, s_{0}$, are arbitrary positive real numbers and $\alpha, \beta, \gamma, \delta$ are positive constants. Also we obtain the form of solution of some special cases of this equation.

Various biological systems naturally leads to their study by means of a discrete variable. Appropriate examples include population dynamics and medicine. Some fundamental models of biological phenomena, including harvesting of fish, a single species population model, ventilation volume and blood $\mathrm{CO} 2$ levels, the production of red blood cells, a simple epidemics model, and a model of waves of disease that can be analyzed by difference equations are shown in [1]. Newly, there has been interest in so-called dynamical diseases, which correspond to physiological disorders for which a generally stable control system becomes unstable. One of the first papers on this subject was that of Mackey and Glass [2]. In which they investigated a first-order difference-delay equation that models the concentration of blood-level CO2. They also discussed models of a second class of diseases associated with the production of red cells, white cells, and platelets in the bone marrow. The dynamical characteristics of population system have been modeled, among others by differential equations in the case of species with overlapping generations and by difference equations in the case of species with non-overlapping generations. In process, one can developed a discrete model directly from observations and experiments. Periodically, for numerical purposes, one wants to propose a finite-difference scheme to numerically solved a given differential equation model, especially when the differential equation cannot be solved explicitly. For a given differential equation, a difference equation approximation would be most acceptable if the solution of the difference equation is the same as the differential equation at the discrete points [3]. But unless we can explicitly solve both equations, it is impossible to satisfy this requirements. Most of the time, it is fascinating that a differential equation, when extracted from a difference equation, marmalade the dynamical features of the corresponding continuous-time model such as equilibria, their local and global stability characteristics, and bifurcation behaviors. If alike discrete models can be derived from continuous time models, and it will preserve the considered realities, such discrete-time models can be called 'dynamically consistent' with the continuous-time models. 
The study of oscillatory and asymptotic stability properties of solution behavior of difference equations is extremely advantageous in the behavior of various biological system and other applications. This is because difference equations are relevant models for expressing situations where the variable is assumed to take only a discrete set of values and they appear frequently in the formulation and analysis of discrete time systems, in the study of biological systems, the study of deterministic chaos, the numerical integration of differential equations by finite difference schemes and so on. Difference equations are good models for describing situations where population growth is not continuous but seasonal with overlapping generations. "For example, the difference equation

$$
\omega_{n+1}=\omega_{n} e^{\left[r\left(1-\frac{\phi_{n}}{k}\right)\right]}
$$

has been expressed to model different animal populations.

The generalized Beverton-Holt stock recruitment model has been investigated in $[4,5]$.

$$
z_{n+1}=A z_{n}+\frac{B z_{n-1}}{1+C z_{n-1}+D z_{n}} .
$$

Several other researchers have studied the behavior of the solution of difference equations for example in [6] E. M. Elsayed investigated the solution of the following non-linear difference equation.

$$
w_{n+1}=a w_{n}+\frac{b w_{n}^{2}}{c w_{n}+d w_{n-1}} .
$$

Elabbasy et al. [7] studied the boundedness, global stability, periodicity character and gave the solution of some special cases of the difference equation.

$$
y_{n+1}=\frac{A y_{n-l}+B y_{n-k}}{\alpha y_{n-l}+\beta y_{n-k}}
$$

Keratas et al. [8] gave the solution of the following difference equation

$$
\ell_{n+1}=\frac{\ell_{n-5}}{1+\ell_{n-2} \ell_{n-5}}
$$

Elabbasy et al. [9] investigated the global stability, periodicity character and gave the solution of some special cases of the difference equation

$$
x_{n+1}=\frac{a x_{n-l} x_{n-k}}{b x_{n-p}+c x_{n-q}} .
$$

Yalçınkaya et al. [10] has studied the following difference equation

$$
x_{n+1}=\alpha+\frac{x_{n-m}}{x_{n}^{k}} .
$$

Saleh et. al. [11] study the solution of difference

$$
y_{n+1}=A+\frac{y_{n}}{y_{n-k}} .
$$

Elsayed et al. [12] studied the global behavior of rational recursive sequence

$$
x_{n+1}=a x_{n-l}+\frac{b x_{n-k}+c x_{n-s}}{d+e x_{n-t}}
$$

As a matter of fact, numerous papers negotiate with the problem of solving nonlinear difference equations in any way possible, see, for instance [4]-[6], [13]-[18]. The long-term behavior and solutions of rational difference equations of order greater than one has been extensively studied during the last decade. For example, various results about periodicity, boundedness, stability, and closed form solution of the second-order rational difference equations have been investigated see [12]-[15], [19]-[25]. Other related work on rational difference equations see in refs. [26]-[28].

Here, we recall some basic definitions and some theorems that we need in the sequel.

\section{Basic definitions}

Let $I$ be some interval of real numbers and let

$$
F: I^{k+1} \rightarrow I
$$

be a continuously differentiable function. Then for every set of initial conditions $s_{-k}, s_{-k+1}, \ldots, s_{0} \in I$, the difference equation

$$
s_{n+1}=F\left(s_{n}, s_{n-1}, \ldots, s_{n-k}\right), n=0,1, \ldots,
$$

has a unique solution $\left\{s_{n}\right\}_{n=-k}^{\infty}$.

A point $\bar{s} \in I$ is called an equilibrium point of 2.1 if

$$
\bar{s}=F(\bar{s}, \bar{s}, \ldots, \bar{s}) .
$$

That is, $s_{n}=\bar{s}$ for $n \geq 0$, is a solution of 2.1 or equivalently $\bar{s}$ is a fixed point of $F$. 
Definition 2.1. (Periodicity) A Sequence $\left\{s_{n}\right\}_{n=-k}^{\infty}$ is said to be periodic with period $p$ if $s_{n+p}=s_{n}$ for all $n \geq-k$.

Definition 2.2. (Fibonacci Sequence). The sequence $\left\{F_{m}\right\}_{m=1}^{\infty}=\{1,2,3,5,8,13, \ldots$.$\} i.e. F_{m}=F_{m-1}+F_{m-2} \geq 0, F_{-2}=0, F_{-1}=1$ is called Fibonacci Sequence.

Definition 2.3. (Stability) (i) The equilibrium point $\bar{s}$ of Eq.(1.2) is locally stable if for every $\varepsilon>0$, there exists $\delta>0$ such that for all $s_{-k}, s_{-k+1}, \ldots, s_{-1}, s_{0} \in I$ with

$$
\left|s_{-k}-\bar{s}\right|+\left|s_{-k+1}-\bar{s}\right|+\ldots+\left|s_{0}-\bar{s}\right|<\delta,
$$

we have

$$
\left|s_{n}-\bar{s}\right|<\varepsilon \quad \text { for all } n \geq-k
$$

(ii) The equilibrium point $\bar{s}$ of 2.1 is locally asymptotically stable if $\bar{s}$ is locally stable solution of Eq.(1.2) and there exists $\gamma>0$, such that for all $s_{-k}, s_{-k+1}, \ldots, s_{-1}, s_{0} \in I$ with

$$
\left|s_{-k}-\bar{s}\right|+\left|s_{-k+1}-\bar{s}\right|+\ldots+\left|s_{0}-\bar{s}\right|<\gamma,
$$

we have

$$
\lim _{n \rightarrow \infty} s_{n}=\bar{s}
$$

(iii) The equilibrium point $\bar{s}$ of 2.1 is global attractor if for all $s_{-k}, s_{-k+1}, \ldots, s_{-1}, s_{0} \in I$, we have

$$
\lim _{n \rightarrow \infty} s_{n}=\bar{s}
$$

(iv) The equilibrium point $\bar{s}$ of 2.1 is globally asymptotically stable if $\bar{s}$ is locally stable, and $\bar{s}$ is also a global attractor of 2.1 .

(v) The equilibrium point $\bar{s}$ of 2.1 is unstable if $\bar{s}$ is not locally stable.

(vi) The linearized equation of 2.1 about the equilibrium $\bar{s}$ is the linear difference equation

$$
y_{n+1}=\sum_{i=0}^{k} \frac{\partial F(\bar{s}, \bar{s}, \ldots, \bar{s})}{\partial s_{n-i}} y_{n-i} .
$$

Theorem 2.4. [2] Assume that $p, q \in R$ and $k \in\{0,1,2, \ldots\}$. Then

$$
|p|+|q|<1
$$

is a sufficient condition for the asymptotic stability of the difference equation

$$
s_{n+1}+p s_{n}+q s_{n-k}=0, \quad n=0,1, \ldots .
$$

Remark 2.5. Theorem 2.4 can be easily extended to a general linear equation of the form

$$
s_{n+k}+p_{1} s_{n+k-1}+\ldots . .+p_{k} s_{n}=0, \quad n=0,1, \ldots
$$

where $p_{1}, p_{2}, \ldots, p_{k} \in R$ and $k \in\{1,2, \ldots\}$. Then 2.2 is asymptotically stable provided that

$$
\sum_{i=1}^{k}\left|p_{i}\right|<1
$$

Consider the following equation

$$
s_{n+1}=g\left(s_{n}, s_{n-1}, s_{n-2}\right) .
$$

The following theorem will be useful for the proof of our results in this paper.

Theorem 2.6. [1] Let $[\alpha, \beta]$ be an interval of real numbers and assume that

$$
g:[\alpha, \beta]^{3} \rightarrow[\alpha, \beta]
$$

is a continuous function satisfying the following properties:

(a) $g(x, y, z)$ is non-decreasing in $x$ and $y \in[\alpha, \beta]$ for each fixed $z \in[\alpha, \beta]$.and $g(x, y, z)$ is non-increasing in $z \in[\alpha, \beta]$ for each fixed $x$ and $y \in[\alpha, \beta]$

(b) If $(\lambda, \mu) \in[\alpha, \beta] \times[\alpha, \beta]$ is a solution of the system

$$
\mu=g(\mu, \mu, \lambda) \quad \text { and } \quad \lambda=g(\lambda, \lambda, \mu),
$$

then $\mu=\lambda$,

and 2.3 has a unique equilibrium $\bar{s} \in[\alpha, \beta]$ and every solution of 2.3 converges to $\bar{s}$." 


\section{Main results}

\subsection{Local stability of the equilibrium point of equation 1.1}

This section elaborates the equilibrium point is local stable. Following relation shows the equilibrium points of 1.1

$$
\bar{s}=\alpha \bar{s}+\frac{\beta \bar{s}^{2}}{\gamma \bar{s}+\delta \bar{s}} .
$$

or

$$
\bar{s}^{2}(1-\alpha)(\gamma+\delta)=\beta \bar{s}^{2}
$$

If $(1-\alpha)(\gamma+\delta) \neq \beta$, then the unique equilibrium point is $\bar{s}=0$

Let $f:(0, \infty)^{3} \longrightarrow(0, \infty)$ be a continuously differentiable function defined by

$$
f(\xi, \eta, \omega)=\alpha \xi+\frac{\beta \xi \eta}{\gamma \eta+\delta \omega} .
$$

Therefore at $\bar{s}=0$

$$
\left(\frac{\partial f}{\partial \xi}\right)_{\bar{s}}=\alpha+\frac{\beta v}{(\gamma \eta+\delta \omega)}, \quad\left(\frac{\partial f}{\partial \eta}\right)_{\bar{s}}=\frac{\beta \delta \xi_{w}}{(\gamma \eta+\delta \omega)^{2}}, \quad\left(\frac{\partial f}{\partial \omega}\right)_{\bar{s}}=\frac{-\beta \delta \xi \eta}{(\gamma \eta+\delta \omega)^{2}}
$$

Then the linearized equation of 1.1 about $\bar{s}$ is

$$
y_{n+1}-\left(\alpha+\frac{\beta v}{(\gamma \eta+\delta \omega)}\right) y_{n}+\left(\frac{\beta \delta u \omega}{(\gamma \eta+\delta \omega)^{2}}\right) y_{n-1}+\left(\frac{-\beta \delta \xi \eta}{(\gamma \eta+\delta \omega)^{2}}\right) y_{n-2}=0 .
$$

Theorem 3.1. The equilibrium point $\bar{s}=0$ of (1) is locally asymptotically stable if $\beta(\gamma+3 \delta)<(\gamma+\delta)^{2}(1-\alpha), \quad \alpha<1$.

Proof. It is follows by Theorem A that 3.2 is asymptotically stable if

$$
\left|\alpha+\frac{\beta}{(\gamma+\delta)}\right|+\left|\frac{\beta \delta}{(\gamma+\delta)^{2}}\right|+\left|\frac{-\beta \delta}{(\gamma+\delta)^{2}}\right|<1,
$$

or

$$
\alpha+\frac{\beta \gamma+3 \beta \delta}{(\gamma+\delta)^{2}}<1
$$

and so

$$
\beta(\gamma+3 \delta)<(\gamma+\delta)^{2}(1-\alpha)
$$

Which completes the proof.

\subsection{Global attractivity of the equilibrium point of equation 1.1}

This section investigate the global attractivity character of solutions of 1.1.

Theorem 3.2. The equilibrium point $\bar{s}$ of 1.1 is global attractor. if

$$
\gamma(1-\alpha) \neq \beta
$$

Proof. Let $\alpha, \beta$ are real numbers and assume that $g:[\alpha, \beta]^{3} \rightarrow[\alpha, \beta]$, be a function defined by $g(u, v, w)=\alpha u+\frac{\beta u v}{\gamma v+\delta w}$ then we can easily see that the function $g(u, v, w)$ is increasing in $u, v$ and decreasing in $w$.

Suppose that $(\lambda, \mu)$ is a solution of the system

$$
\mu=g(\mu, \mu, \lambda) \text { and } \lambda=g(\lambda, \lambda, \mu)
$$

Then from 1.1 we see that

Therefore,

$$
\mu=\alpha \mu+\frac{\beta \mu^{2}}{\gamma \mu+\delta \lambda}, \quad \lambda=\alpha \lambda+\frac{\beta \lambda^{2}}{\gamma \lambda+\delta \mu}
$$

or

$$
\mu(1-\alpha)=\frac{\beta \mu^{2}}{\gamma \mu+\delta \lambda}, \quad \lambda(1-\alpha)=\frac{\beta \lambda^{2}}{\gamma \lambda+\delta \mu}
$$

subtracting

$$
\beta \mu^{2}=\gamma(1-\alpha) \mu^{2}+\delta(1-\alpha) \mu \lambda \quad \text { and } \beta \lambda^{2}=\gamma(1-\alpha) \lambda^{2}+\delta(1-\alpha) \mu \lambda,
$$

Thus

$$
\gamma(1-\alpha)\left(\mu^{2}-\lambda^{2}\right)=\beta\left(\mu^{2}-\lambda^{2}\right), \quad \gamma(1-\alpha) \neq \beta
$$

$\mu=\lambda$.

It follows by the Theorem B that $\bar{x}$ is a global attractor of 1.1 and then the proof is complete. 


\section{Boundedness of solutions of $\mathbf{1 . 1}$}

In this section we study the boundedness of solution of 1.1.

Theorem 4.1. Every solution of 1.1 is bounded if

$$
\left(\alpha+\frac{\beta}{\gamma}\right)<1
$$

Proof. Let $\left\{s_{n}\right\}_{n=-5}^{\infty}$ be a solution of 1.1. It follows from 1.1 that

$$
s_{n+1}=\alpha s_{n}+\frac{\beta s_{n} s_{n-4}}{\gamma s_{n-4}+\delta s_{n-5}} \leq \alpha s_{n}+\frac{\beta s_{n} s_{n-4}}{\gamma s_{n-4}}=\left(\alpha+\frac{\beta}{\gamma}\right) s_{n}
$$

Then

$$
s_{n+1} \leq s_{n}, \text { for all } n \geq 0
$$

Then the sub-sequence $\left\{s_{5 n-1}\right\}_{n=-5}^{\infty},\left\{s_{5 n-2}\right\}_{n=-5}^{\infty},\left\{s_{5 n-3}\right\}_{n=-5}^{\infty},\left\{s_{5 n-4}\right\}_{n=-5}^{\infty}$, and $\left\{s_{5 n-4}\right\}_{n=-5}^{\infty}$ are decreasing and so are bounded from above by $M=\max \left\{s_{-5}, s_{-4}, s_{-3}, s_{-2}, s_{-1}, s_{0}\right\}$

Example 4.2. Let $\alpha=0.03, \beta=0.6, \gamma=0.8, \delta=0.01$ and $\alpha=0.3, \beta=0.06, \gamma=0.7 \delta=0.01$ Then 1.1 in this case will be

$$
\begin{gathered}
s_{n+1}=0.03 s_{n}+\frac{0.6 s_{n} s_{n-4}}{0.8 s_{n-4}+0.01 s_{n-5}} \\
s_{n+1}=0.3 s_{n}+\frac{0.06 s_{n} s_{n-4}}{0.7 s_{n-4}+0.1 s_{n-5}}
\end{gathered}
$$

with initial condition for $4.1 s_{-5}=9, s_{-4}=4, s_{-3}=7, s_{-2}=1, s_{-1}=10, s_{0}=8$. The plot for solution of $s_{n}$ is shown in (Figure 4.1) and for $4.2 s_{-5}=5.2, s_{-4}=2.3, s_{-3}=1.2, s_{-2}=0.07, s_{-1}=0.2, s_{0}=0.01$. The plot for solution of $s_{n}$ is shown in (Figure 4.2.)

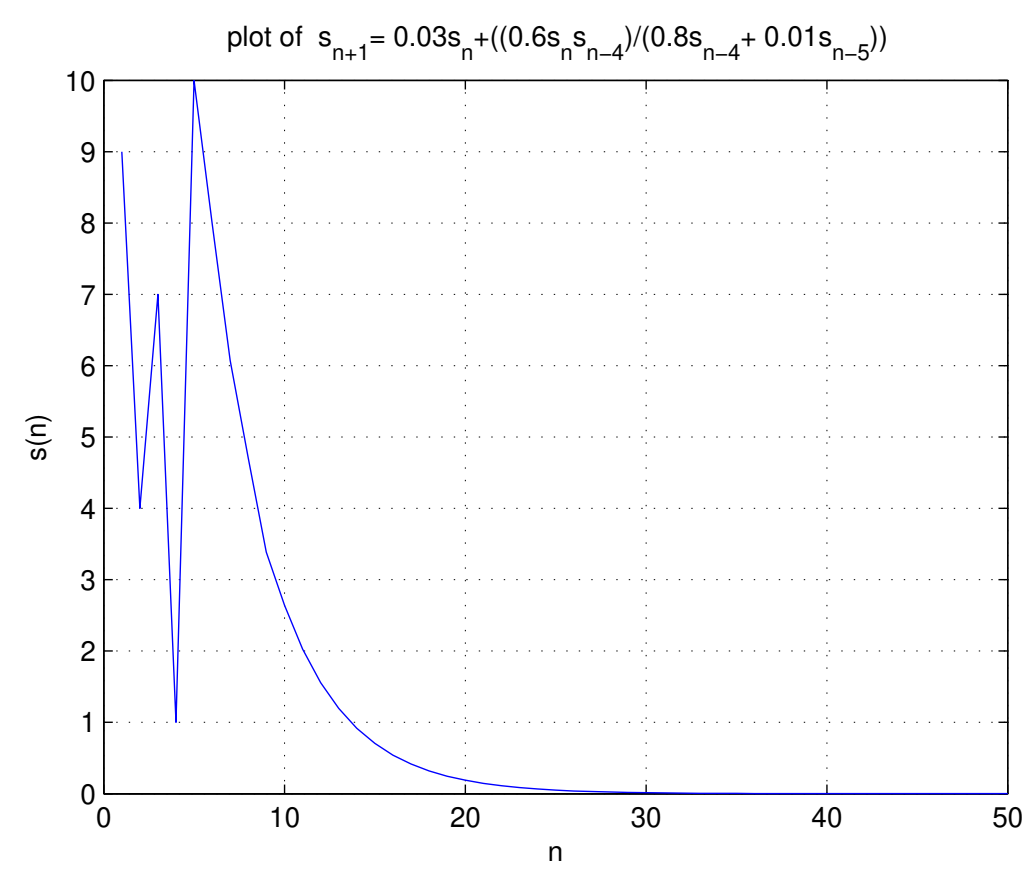

Figure 4.1: Shows Bounded Solution of 4.1 


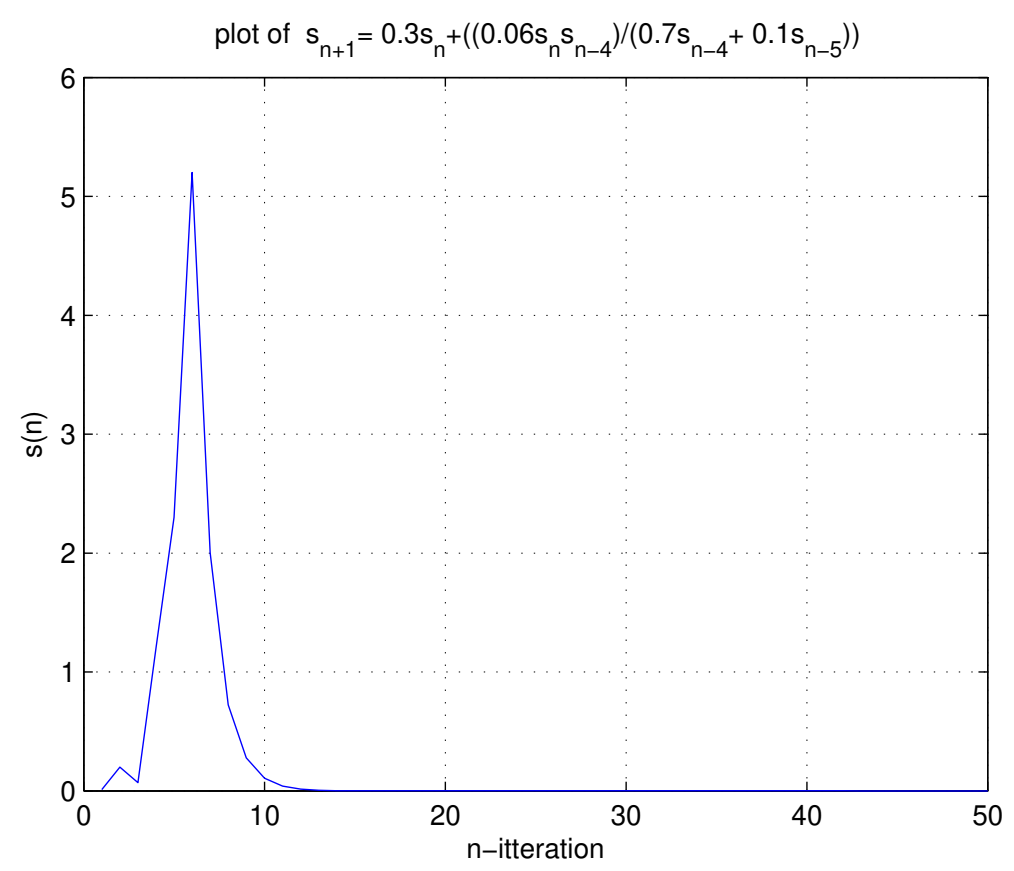

Figure 4.2: Shows Bounded Solution of 4.2

\section{Some special cases of $\mathbf{1 . 1}$}

\subsection{First equation}

Here we will find the closed form expression of solution of special case of 1.1

$$
s_{n+1}=s_{n}+\frac{s_{n} s_{n-4}}{s_{n-4}+s_{n-5}}, \quad n=0,1, \ldots
$$

where the initial conditions $s_{-5}, s_{-4}, s_{-3}, s_{-2}, s_{-1}, s_{0}$ are arbitrary positive real numbers.

Theorem 5.1. Let $\left\{s_{n}\right\}_{n=-5}^{\infty}$ be a solution of 5.1. Then for $n=0,1,2, \ldots$

$$
\begin{aligned}
& s_{5 n}=\varkappa \prod_{i=0}^{n-1}\left(\frac{f_{2 i+3} \varepsilon+f_{2 i+2} \varepsilon}{f_{2 i+2} \varepsilon+f_{2 i+1} \varepsilon}\right)\left(\frac{f_{2 i+3} \rho+f_{2 i+2} \varepsilon}{f_{2 i+2} \rho+f_{2 i+1} \varepsilon}\right)\left(\frac{f_{2 i+3} \imath+f_{2 i+2} \rho}{f_{2 i+2} \imath+f_{2 i+1} \rho}\right)\left(\frac{f_{2 i+3} \sigma+f_{2 i+2} l}{f_{2 i+2} \sigma+f_{2 i+1} l}\right)\left(\frac{f_{2 i+3} \varkappa+f_{2 i+2} \sigma}{f_{2 i+2} \varkappa+f_{2 i+1} \sigma}\right), \\
& s_{5 n+1}=\varkappa \prod_{i=0}^{n}\left(\frac{f_{2 i+3} \varepsilon+f_{2 i+2} \varepsilon}{f_{2 i+2} \varepsilon+f_{2 i+1} \varepsilon}\right)\left(\frac{f_{2 i+1} \rho+f_{2 i} \varepsilon}{f_{2 i} \rho+f_{2 i-1} \varepsilon}\right)\left(\frac{f_{2 i+1} \imath+f_{2 i} \rho}{f_{2 i} \imath+f_{2 i-1} \rho}\right)\left(\frac{f_{2 i+1} \sigma+f_{2 i} \imath}{f_{2 i} \sigma+f_{2 i-1} l}\right)\left(\frac{f_{2 i+1} \varkappa+f_{2 i} \sigma}{f_{2 i} l+f_{2 i-1} \sigma}\right), \\
& s_{5 n+2}=\varkappa \prod_{i=0}^{n}\left(\frac{f_{2 i+3} \varepsilon+f_{2 i+2} \varepsilon}{f_{2 i+2} \varepsilon+f_{2 i+1} \varepsilon}\right)\left(\frac{f_{2 i+3} \rho+f_{2 i+2} \varepsilon}{f_{2 i+2} \rho+f_{2 i+1} \varepsilon}\right)\left(\frac{f_{2 i+1} \iota+f_{2 i} \rho}{f_{2 i} \imath+f_{2 i-1} \rho}\right)\left(\frac{f_{2 i+1} \sigma+f_{2 i} \iota}{f_{2 i} \sigma+f_{2 i-1} l}\right)\left(\frac{f_{2 i+1} \varkappa+f_{2 i} \sigma}{f_{2 i} l+f_{2 i-1} k}\right), \\
& s_{5 n+3}=\varkappa \prod_{i=0}^{n}\left(\frac{f_{2 i+3} \varepsilon+f_{2 i+2} \varepsilon}{f_{2 i+2} \varepsilon+f_{2 i+1} \varepsilon}\right)\left(\frac{f_{2 i+3} \rho+f_{2 i+2} \varepsilon}{f_{2 i+2} \rho+f_{2 i+1} \varepsilon}\right)\left(\frac{f_{2 i+3} \imath+f_{2 i+2} \rho}{f_{2 i+2} \imath+f_{2 i+1} \rho}\right)\left(\frac{f_{2 i+1} \sigma+f_{2 i} \imath}{f_{2 i} \sigma+f_{2 i-1} \imath}\right)\left(\frac{f_{2 i+1} \varkappa+f_{2 i} \sigma}{f_{2 i} \varkappa+f_{2 i-1} \sigma}\right), \\
& s_{5 n+4}=\varkappa \prod_{i=0}^{n}\left(\frac{f_{2 i+3} \varepsilon+f_{2 i+2} \varepsilon}{f_{2 i+2} \varepsilon+f_{2 i+1} \varepsilon}\right)\left(\frac{f_{2 i+3} \rho+f_{2 i+2} \varepsilon}{f_{2 i+2} \rho+f_{2 i+1} \varepsilon}\right)\left(\frac{f_{2 i+3} \imath+f_{2 i+2} \rho}{f_{2 i+2} \imath+f_{2 i+1} \rho}\right)\left(\frac{f_{2 i+3} \sigma+f_{2 i+2} \imath}{f_{2 i+2} \sigma+f_{2 i+1} \imath}\right)\left(\frac{f_{2 i+1} \varkappa+f_{2 i} \sigma}{f_{2 i} \varkappa+f_{2 i-1} \sigma}\right),
\end{aligned}
$$

where $s_{-5}=\varepsilon, s_{-4}=\varepsilon, s_{-3}=\rho, s_{-2}=\imath, s_{-1}=\sigma, s_{0}=\varkappa, \quad\left\{f_{m}\right\}_{m=1}^{\infty}=\{1,1,2,3,5,8,13, \ldots \ldots\}, f_{-1}=f_{0}=1$ 
Proof. For $n=0$ result holds. Now suppose that $n>0$ and that our assumption holds for $n-1, n-2$. That is,

$$
\begin{aligned}
& s_{5 n-6}=\varkappa \prod_{i=0}^{n-2}\left(\frac{f_{2 i+3} \varepsilon+f_{2 i+2} \varepsilon}{f_{2 i+2} \varepsilon+f_{2 i+1} \varepsilon}\right)\left(\frac{f_{2 i+3} \rho+f_{2 i+2} \varepsilon}{f_{2 i+2} \rho+f_{2 i+1} \varepsilon}\right)\left(\frac{f_{2 i+3} \imath+f_{2 i+2} \rho}{f_{2 i+2} \iota+f_{2 i+1} \rho}\right)\left(\frac{f_{2 i+3} \sigma+f_{2 i+2} \iota}{f_{2 i+2} \sigma+f_{2 i+1} \imath}\right)\left(\frac{f_{2 i+1} \varkappa+f_{2 i} \sigma}{f_{2 i} \varkappa+f_{2 i-1} \sigma}\right), \\
& s_{5 n-5}=\varkappa \prod_{i=0}^{n-2}\left(\frac{f_{2 i+3} \varepsilon+f_{2 i+2} \varepsilon}{f_{2 i+2} \varepsilon+f_{2 i+1} \varepsilon}\right)\left(\frac{f_{2 i+3} \rho+f_{2 i+2} \varepsilon}{f_{2 i+2} \rho+f_{2 i+1} \varepsilon}\right)\left(\frac{f_{2 i+3} \iota+f_{2 i+2} \rho}{f_{2 i+2} \iota+f_{2 i+1} \rho}\right)\left(\frac{f_{2 i+3} \sigma+f_{2 i+2} \iota}{f_{2 i+2} \sigma+f_{2 i+1} \iota}\right)\left(\frac{f_{2 i+3} \varkappa+f_{2 i+2} \sigma}{f_{2 i+2} \varkappa+f_{2 i+1} \sigma}\right) \text {, } \\
& s_{5 n-4}=\varkappa \prod_{i=0}^{n-1}\left(\frac{f_{2 i+3} \varepsilon+f_{2 i+2} \varepsilon}{f_{2 i+2} \varepsilon+f_{2 i+1} \varepsilon}\right)\left(\frac{f_{2 i+1} \rho+f_{2 i} \varepsilon}{f_{2 i} \rho+f_{2 i-1} \varepsilon}\right)\left(\frac{f_{2 i+1} \imath+f_{2 i} \rho}{f_{2 i} \iota+f_{2 i-1} \rho}\right)\left(\frac{f_{2 i+1} \sigma+f_{2 i} \imath}{f_{2 i} \sigma+f_{2 i-1} \imath}\right)\left(\frac{f_{2 i+1} \varkappa+f_{2 i} \sigma}{f_{2 i} \varkappa+f_{2 i-1} \sigma}\right), \\
& s_{5 n-3}=\varkappa \prod_{i=0}^{n-1}\left(\frac{f_{2 i+3} \varepsilon+f_{2 i+2} \varepsilon}{f_{2 i+2} \varepsilon+f_{2 i+1} \varepsilon}\right)\left(\frac{f_{2 i+3} \rho+f_{2 i+2} \varepsilon}{f_{2 i+2} \rho+f_{2 i+1} \varepsilon}\right)\left(\frac{f_{2 i+1} \imath+f_{2 i} \rho}{f_{2 i} \imath+f_{2 i-1} \rho}\right)\left(\frac{f_{2 i+1} \sigma+f_{2 i} \iota}{f_{2 i} \sigma+f_{2 i-1} l}\right)\left(\frac{f_{2 i+1} \varkappa+f_{2 i} \sigma}{f_{2 i} \varkappa+f_{2 i-1} \sigma}\right), \\
& s_{5 n-2}=\varkappa \prod_{i=0}^{n-1}\left(\frac{f_{2 i+3} \varepsilon+f_{2 i+2} \varepsilon}{f_{2 i+2} \varepsilon+f_{2 i+1} \varepsilon}\right)\left(\frac{f_{2 i+3} \rho+f_{2 i+2} \varepsilon}{f_{2 i+2} \rho+f_{2 i+1} \varepsilon}\right)\left(\frac{f_{2 i+3} \imath+f_{2 i+2} \rho}{f_{2 i+2} \iota+f_{2 i+1} \rho}\right)\left(\frac{f_{2 i+1} \sigma+f_{2 i} \imath}{f_{2 i} \sigma+f_{2 i-1} \imath}\right)\left(\frac{f_{2 i+1} \varkappa+f_{2 i} \sigma}{f_{2 i} \varkappa+f_{2 i-1} \sigma}\right) \text {, } \\
& s_{5 n-1}=\varkappa \prod_{i=0}^{n-1}\left(\frac{f_{2 i+3} \varepsilon+f_{2 i+2} \varepsilon}{f_{2 i+2} \varepsilon+f_{2 i+1} \varepsilon}\right)\left(\frac{f_{2 i+3} \rho+f_{2 i+2} \varepsilon}{f_{2 i+2} \rho+f_{2 i+1} \varepsilon}\right)\left(\frac{f_{2 i+3} \imath+f_{2 i+2} \rho}{f_{2 i+2} \iota+f_{2 i+1} \rho}\right)\left(\frac{f_{2 i+3} \sigma+f_{2 i+2} \iota}{f_{2 i+2} \sigma+f_{2 i+1} \imath}\right)\left(\frac{f_{2 i+1} \varkappa+f_{2 i} \sigma}{f_{2 i} \varkappa+f_{2 i-1} \sigma}\right) \text {, }
\end{aligned}
$$

Now, we see from 5.1 that

$$
s_{5 n+1}=s_{5 n}+\frac{s_{5 n} s_{5 n-4}}{s_{5 n-4}+s_{5 n-5}}
$$

$=\left(\varkappa \prod_{i=0}^{n-1}\left(\frac{f_{2 i+3} \varepsilon+f_{2 i+2} \varepsilon}{f_{2 i+2} \varepsilon+f_{2 i+1} \varepsilon}\right)\left(\frac{f_{2 i+3} \rho+f_{2 i+2} \varepsilon}{f_{2 i+2} \rho+f_{2 i+1} \varepsilon}\right)\left(\frac{f_{2 i+3} \imath+f_{2 i+2} \rho}{f_{2 i+2} \imath+f_{2 i+1} \rho}\right)\left(\frac{f_{2 i+3} \sigma+f_{2 i+2} \imath}{f_{2 i+2} \sigma+f_{2 i+1} \imath}\right)\left(\frac{f_{2 i+3} \imath+f_{2 i+2} \sigma}{f_{2 i+2} \varkappa+f_{2 i+1} \sigma}\right)\right)$

$\left(\varkappa \prod_{i=0}^{n-1}\left(\frac{f_{2 i+3} \varepsilon+f_{2 i+2} \varepsilon}{f_{2 i+2} \varepsilon+f_{2 i+1} \varepsilon}\right)\left(\frac{f_{2 i+3} \rho+f_{2 i+2} \varepsilon}{f_{2 i+2} \rho+f_{2 i+1} q}\right)\left(\frac{f_{2 i+3} l+f_{2 i+2} \rho}{f_{2 i+2} l+f_{2 i+1} \rho}\right)\left(\frac{f_{2 i+3} \sigma+f_{2 i+2} l}{f_{2 i+2} \sigma+f_{2 i+1} l}\right)\left(\frac{f_{2 i+3} \varkappa+f_{2 i+2} \sigma}{f_{2 i+2} \varkappa+f_{2 i+1} \sigma}\right)\right) \times$

$+\frac{\left(\varkappa \prod_{i=0}^{n-1}\left(\frac{f_{2 i+3} \varepsilon+f_{2 i+2} \varepsilon}{f_{2 i+2} \varepsilon+f_{2 i+1} \varepsilon}\right)\left(\frac{f_{2 i+1} \rho+f_{2 i} \varepsilon}{f_{2 i} \rho+f_{2 i-1} \varepsilon}\right)\left(\frac{f_{2 i+1} l+f_{2 i} p}{f_{2 i} l+f_{2 i-1} \rho}\right)\left(\frac{f_{2 i+1} \sigma+f_{2 i} l}{f_{2 i} \sigma+f_{2 i-1} l}\right)\left(\frac{f_{2 i+1} \varkappa+f_{2 i} \sigma}{f_{2 i} \varkappa+f_{2 i-1} \sigma}\right)\right)}{\left[\begin{array}{c}\varkappa \prod_{i=0}^{n-1}\left(\frac{f_{2 i+3} \varepsilon+f_{2 i+2} \varepsilon}{f_{2 i+2} \varepsilon+f_{2 i+1} \varepsilon}\right)\left(\frac{f_{2 i+3} \rho+f_{2 i+2} \varepsilon}{f_{2 i+2} \rho+f_{2 i+1} \varepsilon}\right)\left(\frac{f_{2 i+3} l+f_{2 i+2} \rho}{f_{2 i+2} l+f_{2 i+1} \rho}\right)\left(\frac{f_{2 i+3} \sigma+f_{2 i+2} l}{f_{2 i+2} \sigma+f_{2 i+1} l}\right)\left(\frac{f_{2 i+3} \varkappa+f_{2 i+2} \sigma}{f_{2 i+2} \varkappa+f_{2 i+1} k}\right) \\ +\varkappa \prod_{i=0}^{n-2}\left(\frac{f_{2 i+3} \varepsilon+f_{2 i+2} \varepsilon}{f_{2 i+2} \varepsilon+f_{2 i+1} \varepsilon}\right)\left(\frac{f_{2 i+3} \rho+f_{2 i+2} \varepsilon}{f_{2 i+2} \rho+f_{2 i+1} \varepsilon}\right)\left(\frac{f_{2 i+3} l+f_{2 i+2} \rho}{f_{2 i+2} l+f_{2 i+1} \rho}\right)\left(\frac{f_{2 i+3} \sigma+f_{2 i+2} l}{f_{2 i+2} \sigma+f_{2 i+1} l}\right)\left(\frac{f_{2 i+3} \varkappa+f_{2 i+2} \sigma}{f_{2 i+2} \varkappa+f_{2 i+1} \sigma}\right)\end{array}\right]}$

$=\varkappa \prod_{i=0}^{n-1}\left(\frac{f_{2 i+3} \varepsilon+f_{2 i+2} \varepsilon}{f_{2 i+2} \varepsilon+f_{2 i+1} \varepsilon}\right)\left(\frac{f_{2 i+3} \rho+f_{2 i+2} \varepsilon}{f_{2 i+2} \rho+f_{2 i+1} \varepsilon}\right)\left(\frac{f_{2 i+3} \iota+f_{2 i+2} \rho}{f_{2 i+2} \iota+f_{2 i+1} \rho}\right)\left(\frac{f_{2 i+3} \sigma+f_{2 i+2} \iota}{f_{2 i+2} \sigma+f_{2 i+1} \iota}\right)\left(\frac{f_{2 i+3} \varkappa+f_{2 i+2} \sigma}{f_{2 i+2} \varkappa+f_{2 i+1} \sigma}\right)$ $+\frac{\varkappa \prod_{i=0}^{n-1}\left(\frac{f_{2 i+3} \varepsilon+f_{2 i+2} \varepsilon}{f_{2 i+2} \varepsilon+f_{2 i+1} \varepsilon}\right)\left(\frac{f_{2 i+3} \rho+f_{2 i+2} \varepsilon}{f_{2 i+2} \rho+f_{2 i+1} \varepsilon}\right)\left(\frac{f_{2 i+3} l+f_{2 i+2} \rho}{f_{2 i+2} l+f_{2 i+1} \rho}\right)\left(\frac{f_{2 i+3} \sigma+f_{2 i+2} l}{f_{2 i+2} \sigma+f_{2 i+1} l}\right)\left(\frac{f_{2 i+3} \varkappa+f_{2 i+2} \sigma}{f_{2 i+2} \varkappa+f_{2 i+1} \sigma}\right)\left(\frac{f_{2 n+1} \varepsilon+f_{2 n} \varepsilon}{f_{2 n} \varepsilon+f_{2 n-1} \varepsilon}\right)}{\left(\frac{f_{2 n+1} \varepsilon+f_{2 n} \varepsilon}{f_{2 n} \varepsilon+f_{2 n-1} \varepsilon}\right)+1}$

$$
=\varkappa \prod_{i=0}^{n-1}\left(\frac{f_{2 i+3} \varepsilon+f_{2 i+2} \varepsilon}{f_{2 i+2} \varepsilon+f_{2 i+1} \varepsilon}\right)\left(\frac{f_{2 i+3} \rho+f_{2 i+2} \varepsilon}{f_{2 i+2} \rho+f_{2 i+1} \varepsilon}\right)\left(\frac{f_{2 i+3} \iota+f_{2 i+2} \rho}{f_{2 i+2} \iota+f_{2 i+1} \rho}\right)\left(\frac{f_{2 i+3} \sigma+f_{2 i+2} \rho}{f_{2 i+2} \sigma+f_{2 i+1} \iota}\right)\left(\frac{f_{2 i+3} \varkappa+f_{2 i+2} \sigma}{f_{2 i+2} \varkappa+f_{2 i+1} \sigma}\right)
$$$$
+\frac{\varkappa \prod_{i=0}^{n-1}\left(\frac{f_{2 i+3} \varepsilon+f_{2 i+2} \varepsilon}{f_{2 i+2} \varepsilon+f_{2 i+1} \varepsilon}\right)\left(\frac{f_{2 i+3} \rho+f_{2 i+2} \varepsilon}{f_{2 i+2} \rho+f_{2 i+1} \varepsilon}\right)\left(\frac{f_{2 i+3} l+f_{2 i+2} \rho}{f_{2 i+2} l+f_{2 i+1} \rho}\right)\left(\frac{f_{2 i+3} \sigma+f_{2 i+2} l}{f_{2 i+2} \sigma+f_{2 i+1} l}\right)\left(\frac{f_{2 i+3} \varkappa+f_{2 i+2} \sigma}{f_{2 i+2} \varkappa+f_{2 i+1} \sigma}\right)\left(f_{2 n} \varepsilon+f_{2 n-1} \varepsilon\right)}{f_{2 n+1} \varepsilon+f_{2 n} \varepsilon+f_{2 n} \varepsilon+f_{2 n-1} \varepsilon}
$$

$=\varkappa \prod_{i=0}^{n-1}\left(\frac{f_{2 i+3} \varepsilon+f_{2 i+2} \varepsilon}{f_{2 i+2} \varepsilon+f_{2 i+1} \varepsilon}\right)\left(\frac{f_{2 i+3} \rho+f_{2 i+2} \varepsilon}{f_{2 i+2} \rho+f_{2 i+1} \varepsilon}\right)\left(\frac{f_{2 i+3} \imath+f_{2 i+2} \rho}{f_{2 i+2} \imath+f_{2 i+1} \rho}\right)\left(\frac{f_{2 i+3} \sigma+f_{2 i+2} \imath}{f_{2 i+2} \sigma+f_{2 i+1} \imath}\right)\left(\frac{f_{2 i+3} \varkappa+f_{2 i+2} \sigma}{f_{2 i+2} \varkappa+f_{2 i+1} \sigma}\right)\left(1+\frac{f_{2 n+1} \varepsilon+f_{2 n} \varepsilon}{f_{2 n+2} \varepsilon+f_{2 n+1} \varepsilon}\right)$

Thus,

$$
s_{5 n+1}=\varkappa \prod_{i=0}^{n}\left(\frac{f_{2 i+3} \varepsilon+f_{2 i+2} \varepsilon}{f_{2 i+2} \varepsilon+f_{2 i+1} \varepsilon}\right)\left(\frac{f_{2 i+1} \rho+f_{2 i} \varepsilon}{f_{2 i} \rho+f_{2 i-1} \varepsilon}\right)\left(\frac{f_{2 i+1} \imath+f_{2 i} \rho}{f_{2 i} \imath+f_{2 i-1} \rho}\right)\left(\frac{f_{2 i+1} \sigma+f_{2 i} \iota}{f_{2 i} \sigma+f_{2 i-1} \imath}\right)\left(\frac{f_{2 i+1} \varkappa+f_{2 i} \sigma}{f_{2 i} \varkappa+f_{2 i-1} \sigma}\right)
$$

Again, it follows from equation 5.1 that,

$$
s_{5 n+3}=s_{5 n+2}+\frac{s_{5 n+2} s_{5 n-2}}{s_{5 n-2}+s_{5 n-3}}
$$




$$
\begin{aligned}
& =\varkappa \prod_{i=0}^{n}\left(\frac{f_{2 i+3} \varepsilon+f_{2 i+2} \varepsilon}{f_{2 i+2} \varepsilon+f_{2 i+1} \varepsilon}\right)\left(\frac{f_{2 i+3} \rho+f_{2 i+2} \varepsilon}{f_{2 i+2} \rho+f_{2 i+1} \varepsilon}\right)\left(\frac{f_{2 i+1} \imath+f_{2 i} \rho}{f_{2 i} \imath+f_{2 i-1} \rho}\right)\left(\frac{f_{2 i+1} \sigma+f_{2 i} \iota}{f_{2 i} \sigma+f_{2 i-1} \imath}\right)\left(\frac{f_{2 i+1} \varkappa+f_{2 i} \sigma}{f_{2 i} \varkappa+f_{2 i-1} \sigma}\right) \\
& \left(\varkappa \prod_{i=0}^{n}\left(\frac{f_{2 i+3} \varepsilon+f_{2 i+2} \varepsilon}{f_{2 i+2} \varepsilon+f_{2 i+1} \varepsilon}\right)\left(\frac{f_{2 i+3} \rho+f_{2 i+2} \varepsilon}{f_{2 i+2} \rho+f_{2 i+1} \varepsilon}\right)\left(\frac{f_{2 i+1} l+f_{2 i} \rho}{f_{2 i} l+f_{2 i-1} \rho}\right)\left(\frac{f_{2 i+1} \sigma+f_{2 i} l}{f_{2 i} \sigma+f_{2 i-1} l}\right)\left(\frac{f_{2 i+1} \varkappa+f_{2 i} \sigma}{f_{2 i} \varkappa+f_{2 i-1} \sigma}\right)\right) \times
\end{aligned}
$$

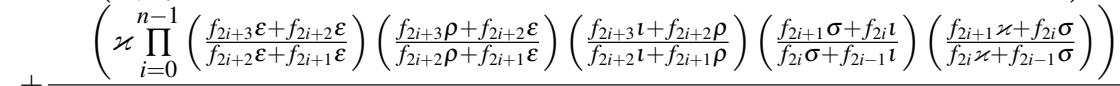

$$
\begin{aligned}
& \left.\begin{array}{l}
\left(\varkappa \prod_{i=0}^{n-1}\left(\frac{f_{2 i+3} \varepsilon+f_{2 i+2} \varepsilon}{f_{2 i+2} \varepsilon+f_{2 i+1} \varepsilon}\right)\left(\frac{f_{2 i+3} \rho+f_{2 i+2} \varepsilon}{f_{2 i+2} \rho+f_{2 i+1} \varepsilon}\right)\left(\frac{f_{2 i+3} l+f_{2 i+2} \rho}{f_{2 i+2} l+f_{2 i+1} \rho}\right)\left(\frac{f_{2 i+1} \sigma+f_{2 i} l}{f_{2 i} \sigma+f_{2 i-1} l}\right)\left(\frac{f_{2 i+1} \varkappa+f_{2 i} \sigma}{f_{2 i} \varkappa+f_{2 i-1} \sigma}\right)\right) \\
+\left(\varkappa \prod_{i=0}^{n-1}\left(\frac{f_{2 i+3} \varepsilon+f_{2 i+2} \varepsilon}{f_{2 i+2} \varepsilon+f_{2 i+1} \varepsilon}\right)\left(\frac{f_{2 i+3} \rho+f_{2 i+2} \varepsilon}{f_{2 i+2} \rho+f_{2 i+1} \varepsilon}\right)\left(\frac{f_{2 i+1} l+f_{2 i} \rho}{f_{2 i} l+f_{2 i-1} \rho}\right)\left(\frac{f_{2 i+1} \sigma+f_{2 i} l}{f_{2 i} \sigma+f_{2 i-1} l}\right)\left(\frac{f_{2 i+1} \varkappa+f_{2 i} \sigma}{f_{2 i} \varkappa+f_{2 i-1} \sigma}\right)\right)
\end{array}\right] \\
& =\prod_{i=0}^{n}\left(\frac{f_{2 i+3} \varepsilon+f_{2 i+2} \varepsilon}{f_{2 i+2} \varepsilon+f_{2 i+1} \varepsilon}\right)\left(\frac{f_{2 i+3} \rho+f_{2 i+2} \varepsilon}{f_{2 i+2} \rho+f_{2 i+1} \varepsilon}\right)\left(\frac{f_{2 i+1} \imath+f_{2 i} \rho}{f_{2 i} \imath+f_{2 i-1} \rho}\right)\left(\frac{f_{2 i+1} \sigma+f_{2 i} \iota}{f_{2 i} \sigma+f_{2 i-1} \imath}\right)\left(\frac{f_{2 i+1} \varkappa+f_{2 i} \sigma}{f_{2 i} \varkappa+f_{2 i-1} \sigma}\right) \\
& +\left[\frac{\varkappa \prod_{i=0}^{n}\left(\frac{f_{2 i+3} \varepsilon+f_{2 i+2} \varepsilon}{f_{2 i+2} \varepsilon+f_{2 i+1} \varepsilon}\right)\left(\frac{f_{2 i+3} \rho+f_{2 i+2} \varepsilon}{f_{2 i+2} \rho+f_{2 i+1} \varepsilon}\right)\left(\frac{f_{2 i+1} l+f_{2 i} \rho}{f_{2 i} l+f_{2 i-1} \rho}\right)\left(\frac{f_{2 i+1} \sigma+f_{2 i} l}{f_{2 i} \sigma+f_{2 i-1} l}\right)\left(\frac{f_{2 i+1} \varkappa+f_{2 i} \sigma}{f_{2 i} \varkappa+f_{2 i-1} \sigma}\right)\left(\frac{f_{2 n+1} l+f_{2 n} \rho}{f_{2 n} l+f_{2 n-1} \rho}\right)}{\left(\frac{f_{2 n+1} l+f_{2 n} \rho}{f_{2 n} l+f_{2 n-1} \rho}\right)+1}\right] \\
& =\varkappa \prod_{i=0}^{n}\left[\left(\frac{f_{2 i+3} \varepsilon+f_{2 i+2} \varepsilon}{f_{2 i+2} \varepsilon+f_{2 i+1} \varepsilon}\right)\left(\frac{f_{2 i+3} \rho+f_{2 i+2} \varepsilon}{f_{2 i+2} \rho+f_{2 i+1} \varepsilon}\right)\left(\frac{f_{2 i+1} \imath+f_{2 i} \rho}{f_{2 i} \boldsymbol{l}+f_{2 i-1} \rho}\right)\left(\frac{f_{2 i+1} \sigma+f_{2 i} \boldsymbol{l}}{f_{2 i} \sigma+f_{2 i-1} \imath}\right)\left(\frac{f_{2 i+1} \varkappa+f_{2 i} \sigma}{f_{2 i} \varkappa+f_{2 i-1} \sigma}\right)\left(1+\frac{f_{2 n+1} \imath+f_{2 n} \rho}{f_{2 n+1} \imath+f_{2 n} \rho+f_{2 n} \imath+f_{2 n-1} \rho}\right)\right] \\
& =\varkappa \prod_{i=0}^{n}\left[\left(\frac{f_{2 i+3} \varepsilon+f_{2 i+2} \varepsilon}{f_{2 i+2} \varepsilon+f_{2 i+1} \varepsilon}\right)\left(\frac{f_{2 i+3} \rho+f_{2 i+2} \varepsilon}{f_{2 i+2} \rho+f_{2 i+1} \varepsilon}\right)\left(\frac{f_{2 i+1} \imath+f_{2 i} \rho}{f_{2 i} \iota+f_{2 i-1} \rho}\right)\left(\frac{f_{2 i+1} \sigma+f_{2 i} \boldsymbol{l}}{f_{2 i} \sigma+f_{2 i-1} \boldsymbol{l}}\right)\left(\frac{f_{2 i+1} \varkappa+f_{2 i} \sigma}{f_{2 i} \varkappa+f_{2 i-1} \sigma}\right)\left(1+\frac{f_{2 n+1} \imath+f_{2 n} \rho}{f_{2 n+2} \imath+f_{2 n+1} \rho}\right)\right]
\end{aligned}
$$

Therefore

$$
s_{5 n+3}=\varkappa \prod_{i=0}^{n}\left(\frac{f_{2 i+3} \varepsilon+f_{2 i+2} \varepsilon}{f_{2 i+2} \varepsilon+f_{2 i+1} \varepsilon}\right)\left(\frac{f_{2 i+3} \rho+f_{2 i+2} \varepsilon}{f_{2 i+2} \rho+f_{2 i+1} \varepsilon}\right)\left(\frac{f_{2 i+3} \imath+f_{2 i+2} \rho}{f_{2 i+2} \imath+f_{2 i+1} \rho}\right)\left(\frac{f_{2 i+1} \sigma+f_{2 i} \imath}{f_{2 i} \sigma+f_{2 i-1} \imath}\right)\left(\frac{f_{2 i+1} \varkappa+f_{2 i} \sigma}{f_{2 i} \varkappa+f_{2 i-1} \sigma}\right) .
$$

Other relations can be done similarly. So, the proof is completed.

Example 5.2. To confirm the result in this case we consider numerical example. Let $\alpha=1, \beta=1, \gamma=1, \delta=1$. Then 1.1 in this case will be

$$
s_{n+1}=s_{n}+\frac{s_{n} s_{n-4}}{s_{n-4}+s_{n-5}}
$$

with initial condition $s_{-5}=2, s_{-4}=8, s_{-3}=5, s_{-2}=3, s_{-1}=1, s_{0}=6$. The plot for solution of $s_{n}$ is shown in Figure 5.1.)

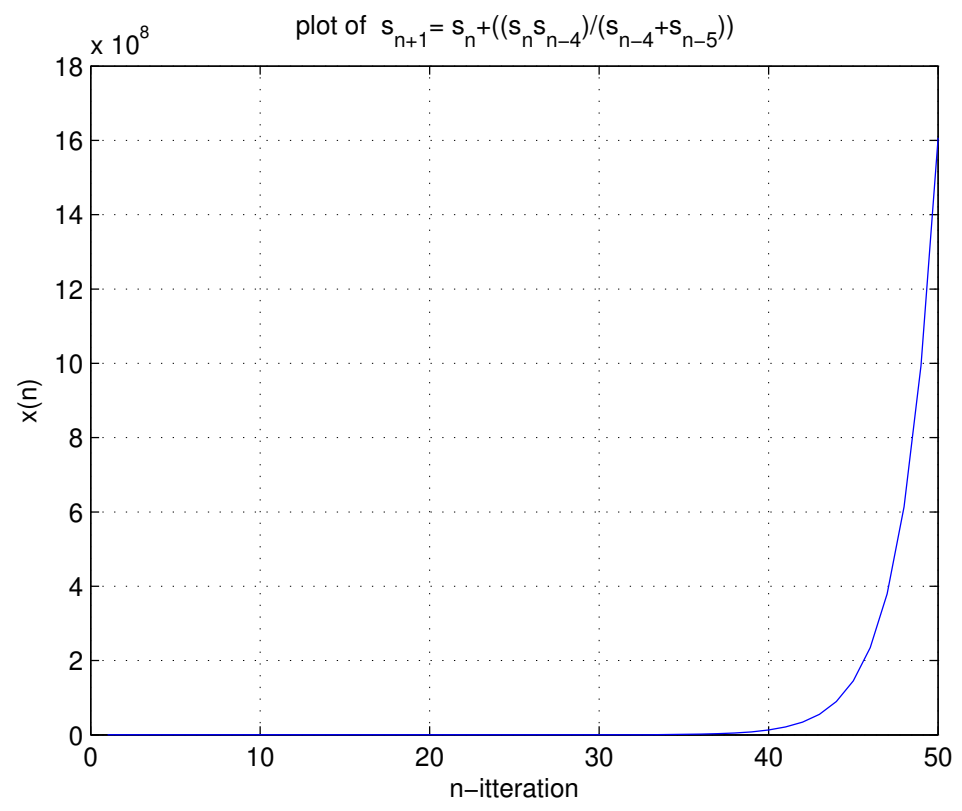

Figure 5.1: Shows Unbounded Solution of 5.2 


\subsection{Second equation}

In this section we solve the specific form of the 1.1

$$
s_{n+1}=s_{n}+\frac{s_{n} s_{n-4}}{s_{n-4}-s_{n-5}}, \quad n=0,1, \ldots
$$

where the initial conditions $s_{-5}, s_{-4}, s_{-3}, s_{-2}, s_{-1}, s_{0}$ are arbitrary positive real numbers.

Theorem 5.3. Let $\left\{s_{n}\right\}_{n=-5}^{\infty}$ be a solution of 5.3. Then for $n=0,1,2, \ldots$.

$$
\begin{gathered}
s_{5 n}=\vartheta \prod_{i=0}^{n-1}\left(\frac{f_{i+3} \sigma-f_{i+1} \omega}{f_{i+1} \sigma-f_{i-1} \omega}\right)\left(\frac{f_{i+3} \rho-f_{i+1} \sigma}{f_{i+1} \rho-f_{i-1} \sigma}\right)\left(\frac{f_{i+3} r-f_{i+1} \rho}{f_{i+1} r-f_{i-1} \rho}\right)\left(\frac{f_{i+3} k-f_{i+1} r}{f_{i+1} k-f_{i-1} r}\right)\left(\frac{f_{i+3} \vartheta-f_{i+1} k}{f_{i+1} \vartheta-f_{i-1} k}\right), \\
s_{5 n+1}=\vartheta\left(\frac{2 \sigma-\omega}{\sigma-\omega}\right) \prod_{i=0}^{n-1}\left(\frac{f_{i+4} \sigma-f_{i+2} \omega}{f_{i+2} \sigma-f_{i} \omega}\right)\left(\frac{f_{i+3} \rho-f_{i+1} \sigma}{f_{i+1} \rho-f_{i-1} \sigma}\right)\left(\frac{f_{i+3} r-f_{i+1} \rho}{f_{i+1} r-f_{i-1} \rho}\right)\left(\frac{f_{i+3} k-f_{i+1} r}{f_{i+1} k-f_{i-1} r}\right)\left(\frac{f_{i+3} \vartheta-f_{i+1} k}{f_{i+1} \vartheta-f_{i-1} k}\right), \\
s_{5 n+2}=\vartheta\left(\frac{2 \sigma-\omega}{\sigma-\omega}\right)\left(\frac{2 \rho-\sigma}{\rho-\sigma}\right) \prod_{i=0}^{n-1}\left(\frac{f_{i+4} \sigma-f_{i+2} \omega}{f_{i+2} \sigma-f_{i} \omega}\right)\left(\frac{f_{i+4} \rho-f_{i+2} \sigma}{f_{i+2} \rho-f_{i} \sigma}\right)\left(\frac{f_{i+3} r-f_{i+1} \rho}{f_{i+1} r-f_{i-1} \rho}\right)\left(\frac{f_{i+3} k-f_{i+1} r}{f_{i+1} k-f_{i-1} r}\right)\left(\frac{f_{i+3} \vartheta-f_{i+1} k}{f_{i+1} \vartheta-f_{i-1} k}\right), \\
s_{5 n+3}=\vartheta\left(\frac{2 \sigma-\omega}{\sigma-\omega}\right)\left(\frac{2 \rho-\sigma}{\rho-\sigma}\right)\left(\frac{2 r-\rho}{r-\rho}\right) \prod_{i=0}^{n-1}\left(\frac{f_{i+4} \sigma-f_{i+2} \omega}{f_{i+2} \sigma-f_{i} \omega}\right)\left(\frac{f_{i+4} \rho-f_{i+2} \sigma}{f_{i+2} \rho-f_{i} \sigma}\right)\left(\frac{f_{i+4} r-f_{i+2} \rho}{f_{i+2} r-f_{i} \rho}\right)\left(\frac{f_{i+3} k-f_{i+1} r}{f_{i+1} k-f_{i-1} r}\right)\left(\frac{f_{i+3} \vartheta-f_{i+1} k}{f_{i+1} \vartheta-f_{i-1} k}\right), \\
s_{5 n+4}=\vartheta\left(\frac{2 \sigma-\omega}{\sigma-\omega}\right)\left(\frac{2 \rho-\sigma}{\rho-\sigma}\right)\left(\frac{2 r-\rho}{r-\rho}\right)\left(\frac{2 k-r}{k-r}\right) \prod_{i=0}^{n-1}\left(\frac{f_{i+4} \sigma-f_{i+2} \omega}{f_{i+2} \sigma-f_{i} \omega}\right)\left(\frac{f_{i+4} \rho-f_{i+2} \sigma}{f_{i+2} \rho-f_{i} \sigma}\right)\left(\frac{f_{i+4} r-f_{i+2} \rho}{f_{i+2} r-f_{i} \rho}\right)\left(\frac{f_{i+4} k-f_{i+2} r}{f_{i+2} k-f_{i} r}\right)\left(\frac{f_{i+3} \vartheta-f_{i+1} k}{f_{i+1} \vartheta-f_{i-1} k}\right), \\
\text { where } s_{-5}=\omega, s_{-4}=\sigma, s_{-3}=\rho, s_{-2}=r, s_{-1}=k, s_{0}=\vartheta,\left\{f_{m}\right\}_{m=1}^{\infty}=\{1,1,2,3,5,8,13, \ldots \ldots . .\}, f_{-1}=f_{0}=1
\end{gathered}
$$

Proof. Same as the Theorem 5.1 and is omitted.

Example 5.4. We will confirm our result by considering some numerical examples. Assume $s_{-5}=1, s_{-4}=3, s_{-3}=2, s_{-2}=9, s_{-1}=$ $6, s_{0}=7$ (see Figure 5.2) and $s_{-5}=13, s_{-4}=12, s_{-3}=18, s_{-2}=16, s_{-1}=15, s_{0}=10$ (see behavior of solution of 5.3 Figure 5.3 ).

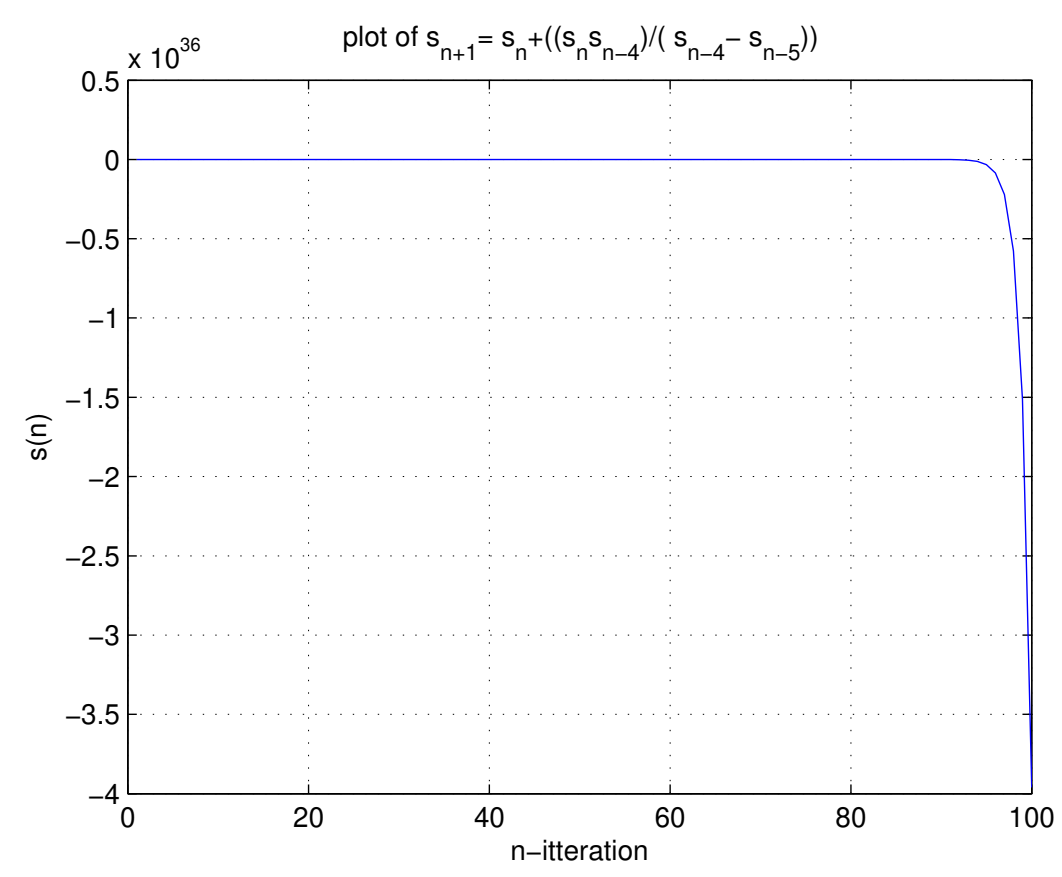

Figure 5.2 


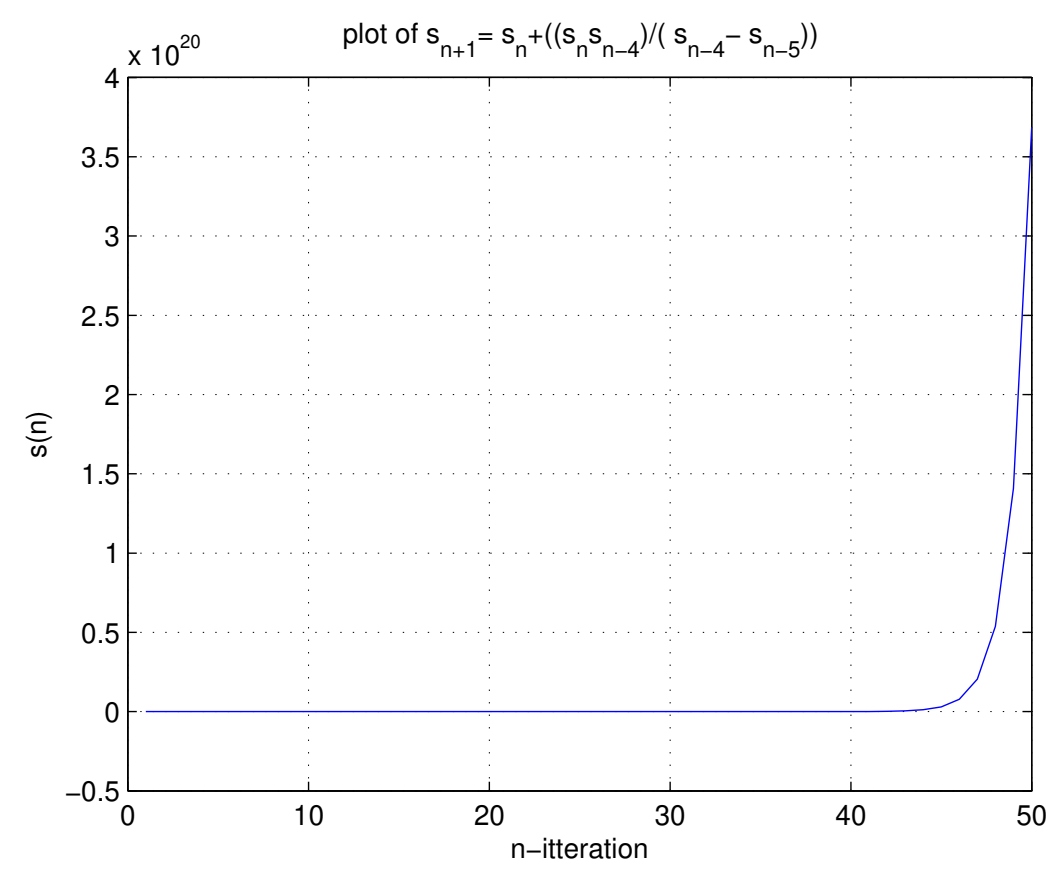

Figure 5.3

\subsection{Third equation}

In this section we deal with the specific form of the 1.1

$$
s_{n+1}=s_{n}-\frac{s_{n} s_{n-4}}{s_{n-4}+s_{n-5}}, \quad n=0,1, \ldots
$$

where the initial conditions $s_{-5}, s_{-4}, s_{-3}, s_{-2}, s_{-1}, s_{0}$ are arbitrary positive real numbers.

Theorem 5.5. $\operatorname{Let}\left\{s_{n}\right\}_{n=-5}^{\infty}$ be a solution of 5.4. Then for $n=0,1,2, \ldots$

$$
\begin{gathered}
s_{5 n}=\frac{l k r p q t}{\left(f_{n} q+f_{n+1} t\right)\left(f_{n} p+f_{n+1} q\right)\left(f_{n} r+f_{n+1} p\right)\left(f_{n} k+f_{n+1} r\right)\left(f_{n} l+f_{n+1} k\right)}, \\
s_{5 n+1}=\frac{l k r p q t}{\left(f_{n+1} q+f_{n+2} t\right)\left(f_{n} p+f_{n+1} q\right)\left(f_{n} r+f_{n+1} p\right)\left(f_{n} k+f_{n+1} r\right)\left(f_{n} l+f_{n+1} k\right)}, \\
s_{5 n+2}=\frac{l k r p q t}{\left(f_{n+1} q+f_{n+2} t\right)\left(f_{n+1} p+f_{n+2} q\right)\left(f_{n} r+f_{n+1} p\right)\left(f_{n} k+f_{n+1} r\right)\left(f_{n} l+f_{n+1} k\right)}, \\
s_{5 n+3}=\frac{l k r p q t}{\left(f_{n+1} q+f_{n+2} t\right)\left(f_{n+1} p+f_{n+2} q\right)\left(f_{n+1} r+f_{n+2} p\right)\left(f_{n} k+f_{n+1} r\right)\left(f_{n} l+f_{n+1} k\right)}, \\
s_{5 n+4}=\frac{l k r p q t}{\left(f_{n+1} q+f_{n+2} t\right)\left(f_{n+1} p+f_{n+2} q\right)\left(f_{n+1} r+f_{n+2} p\right)\left(f_{n+1} k+f_{n+2} r\right)\left(f_{n} l+f_{n+1} k\right)} .
\end{gathered}
$$

Where $s_{-5}=t, s_{-4}=q, s_{-3}=p, s_{-2}=r, s_{-1}=k, s_{0}=l, \quad\left\{f_{m}\right\}_{m=1}^{\infty}=\{1,1,2,3,5,8,13, \ldots \ldots\} \quad f_{0}=1$.

Proof. For $n=0$, the result holds. Now suppose that $n>0$ and that our supposition holds for $n-1, n-2$. That is

$$
\begin{gathered}
s_{5 n-6}=\frac{l k r p q t}{\left(f_{n-1} q+f_{n} t\right)\left(f_{n-1} p+f_{n} q\right)\left(f_{n-1} r+f_{n} p\right)\left(f_{n-1} k+f_{n} r\right)\left(f_{n-2} l+f_{n-1} k\right)}, \\
s_{5 n-5}=\frac{l k r p q t}{\left(f_{n-1} q+f_{n} t\right)\left(f_{n-1} p+f_{n} q\right)\left(f_{n-1} r+f_{n} p\right)\left(f_{n-1} k+f_{n} r\right)\left(f_{n-1} l+f_{n} k\right)}, \\
s_{5 n-4}=\frac{l k r p q t}{\left(f_{n} q+f_{n-1} t\right)\left(f_{n} p+f_{n+1} q\right)\left(f_{n} r+f_{n+1} p\right)\left(f_{n} k+f_{n+1} r\right)\left(f_{n} l+f_{n+1} k\right)},
\end{gathered}
$$




$$
\begin{aligned}
& s_{5 n-3}=\frac{l k r p q t}{\left(f_{n} q+f_{n+1} t\right)\left(f_{n} p+f_{n+1} q\right)\left(f_{n-1} r+f_{n} p\right)\left(f_{n-1} k+f_{n} r\right)\left(f_{n-1} l+f_{n} k\right)}, \\
& s_{5 n-2}=\frac{l k r p q t}{\left(f_{n} q+f_{n+1} t\right)\left(f_{n} p+f_{n+1} q\right)\left(f_{n} r+f_{n+1} p\right)\left(f_{n-1} k+f_{n} r\right)\left(f_{n-1} l+f_{n} k\right)}, \\
& s_{5 n-1}=\frac{l k r p q t}{\left(f_{n} q+f_{n+1} t\right)\left(f_{n} p+f_{n+1} q\right)\left(f_{n} r+f_{n+1} p\right)\left(f_{n} k+f_{n+1} r\right)\left(f_{n-1} l+f_{n} k\right)},
\end{aligned}
$$

Now, from equation 5.1, we see that,

$$
\begin{aligned}
& s_{5 n}=s_{5 n-1}-\frac{s_{5 n-1} s_{5 n-5}}{s_{5 n-5}+s_{5 n-6}} \\
& =\frac{l k r p q t}{\left(f_{n} q+f_{n+1} t\right)\left(f_{n} p+f_{n+1} q\right)\left(f_{n} r+f_{n+1} p\right)\left(f_{n} k+f_{n+1} r\right)\left(f_{n-1} l+f_{n} k\right)} \\
& -\frac{\left(\frac{l k r p q t}{\left(\frac{1}{\left(f_{n} q+f_{n+1} t\right)\left(f_{n} p+f_{n+1} q\right)\left(f_{n} r+f_{n+1} p\right)\left(f_{n} k+f_{n+1} r\right)\left(f_{n-1} l+f_{n} k\right)}\right)}\right)}{\left[\begin{array}{l}
\text { lkrpqt } \\
\left(f_{n-1} q+f_{n} t\right)\left(f_{n-1} p+f_{n} q\right)\left(f_{n-1} r+f_{n} p\right)\left(f_{n-1} k+f_{n} r\right)\left(f_{n-1} l+f_{n} k\right)
\end{array}\right)} \\
& =\frac{l k r p q t}{\left(f_{n} q+f_{n+1} t\right)\left(f_{n} p+f_{n+1} q\right)\left(f_{n} r+f_{n+1} p\right)\left(f_{n} k+f_{n+1} r\right)\left(f_{n-1} l+f_{n} k\right)} \\
& -\frac{\frac{l k r p q t}{\left(f_{n} q+f_{n+1} t\right)\left(f_{n} p+f_{n+1} q\right)\left(f_{n} r+f_{n+1} p\right)\left(f_{n} k+f_{n+1} r\right)\left(f_{n-1} l+f_{n} k\right)}\left(\frac{1}{f_{n-1} l+f_{n} k}\right)}{\left[\frac{1}{\left(f_{n-1} l+f_{n} k\right)}+\frac{1}{\left(f_{n-2} l+f_{n-1} k\right)}\right]} \\
& =\frac{l k r p q t}{\left(f_{n} q+f_{n+1} t\right)\left(f_{n} p+f_{n+1} q\right)\left(f_{n} r+f_{n+1} p\right)\left(f_{n} k+f_{n+1} r\right)\left(f_{n-1} l+f_{n} k\right)} \\
& -\left[\frac{\left(\frac{l k r p q t}{\left(f_{n} q+f_{n+1} t\right)\left(f_{n} p+f_{n+1} q\right)\left(f_{n} r+f_{n+1} p\right)\left(f_{n} k+f_{n+1} r\right)\left(f_{n-1} l+f_{n} k\right)}\right)\left(f_{n-2} l+f_{n-1} k\right)}{f_{n-1} l+f_{n} k+f_{n-2} l+f_{n-1} k}\right] \\
& =\frac{l k r p q t}{\left(f_{n} q+f_{n+1} t\right)\left(f_{n} p+f_{n+1} q\right)\left(f_{n} r+f_{n+1} p\right)\left(f_{n} k+f_{n+1} r\right)\left(f_{n-1} l+f_{n} k\right)}\left(1-\frac{f_{n-2} l+f_{n-1} k}{f_{n} l+f_{n+1} k}\right) \\
& =\frac{l k r p q t}{\left(f_{n} q+f_{n+1} t\right)\left(f_{n} p+f_{n+1} q\right)\left(f_{n} r+f_{n+1} p\right)\left(f_{n} k+f_{n+1} r\right)\left(f_{n-1} l+f_{n} k\right)}\left(\frac{f_{n-1} l+f_{n} k}{f_{n} l+f_{n+1} k}\right)
\end{aligned}
$$

Therefore,

$$
s_{5 n}=\frac{l k r p q t}{\left(f_{n} q+f_{n+1} t\right)\left(f_{n} p+f_{n+1} q\right)\left(f_{n} r+f_{n+1} p\right)\left(f_{n} k+f_{n+1} r\right)\left(f_{n} l+f_{n+1} k\right)}
$$

Now, from equation 5.4

$$
\begin{aligned}
& s_{5 n+4}=s_{5 n+3}-\frac{s_{5 n+3} s_{5 n-1}}{s_{5 n-1}+s_{5 n-2}} \\
& =\frac{l k r p q t}{\left(f_{n+1} q+f_{n+2} t\right)\left(f_{n+1} p+f_{n+2} q\right)\left(f_{n+1} r+f_{n+2} p\right)\left(f_{n} k+f_{n+1} r\right)\left(f_{n} l+f_{n+1} k\right)} \\
& -\left[\begin{array}{c}
\left(\frac{\text { lkrpqt }}{\left(f_{n+1} q+f_{n+2} t\right)\left(f_{n+1} p+f_{n+2} q\right)\left(f_{n+1} r+f_{n+2} p\right)\left(f_{n} k+f_{n+1} r\right)\left(f_{n} l+f_{n+1} k\right)}\right) \\
\left(\frac{l k r p q t}{\left(f_{n} q+f_{n+1} t\right)\left(f_{n} p+f_{n+1} q\right)\left(f_{n} r+f_{n+1} p\right)\left(f_{n} k+f_{n+1} r\right)\left(f_{n-1} l+f_{n} k\right)}\right)
\end{array}\right] \\
& {\left[\begin{array}{c}
\frac{l k r p q t}{\left(f_{n} q+f_{n+1} t\right)\left(f_{n} p+f_{n+1} q\right)\left(f_{n} r+f_{n+1} p\right)\left(f_{n} k+f_{n+1} r\right)\left(f_{n-1} l+f_{n} k\right)} \\
+\frac{l k r p q t}{\left(f_{n} q+f_{n+1} t\right)\left(f_{n} p+f_{n+1} q\right)\left(f_{n} r+f_{n+1} p\right)\left(f_{n-1} k+f_{n} r\right)\left(f_{n-1} l+f_{n} k\right)}
\end{array}\right]}
\end{aligned}
$$




$$
\begin{aligned}
& =\frac{\left(\frac{l k r p q t}{\left(f_{n+1} q+f_{n+2} t\right)\left(f_{n+1} p+f_{n+2} q\right)\left(f_{n+1} r+f_{n+2} p\right)\left(f_{n} k+f_{n+1} r\right)\left(f_{n} l+f_{n+1} k\right)}\right)\left(\frac{1}{f_{n} k+f_{n+1} r}\right)}{\left[\frac{1}{f_{n} k+f_{n+1} r}+\frac{1}{f_{n-1} k+f_{n} r}\right]} \\
& =\frac{l k r p q t}{\left(f_{n+1} q+f_{n+2} t\right)\left(f_{n+1} p+f_{n+2} q\right)\left(f_{n+1} r+f_{n+2} p\right)\left(f_{n} k+f_{n+1} r\right)\left(f_{n} l+f_{n+1} k\right)}\left[1-\frac{f_{n-1} k+f_{n} r}{\left(f_{n-1} k+f_{n} r\right)+\left(f_{n} k+f_{n+1} r\right)}\right] \\
& =\frac{l k r p q t}{\left(f_{n+1} q+f_{n+2} t\right)\left(f_{n+1} p+f_{n+2} q\right)\left(f_{n+1} r+f_{n+2} p\right)\left(f_{n} k+f_{n+1} r\right)\left(f_{n} l+f_{n+1} k\right)}\left[1-\frac{f_{n-1} k+f_{n} r}{f_{n+1} k+f_{n+2} r}\right]
\end{aligned}
$$

Therefore,

$$
s_{5 n+4}=\frac{l k r p q t}{\left(f_{n+1} q+f_{n+2} t\right)\left(f_{n+1} p+f_{n+2} q\right)\left(f_{n+1} r+f_{n+2} p\right)\left(f_{n+1} k+f_{n+2} r\right)\left(f_{n} l+f_{n+1} k\right)} .
$$

Remaining relations can be found similarly. Hence, the proof is completed.

Example 5.6. Assume $s_{-5}=1, s_{-4}=3, s_{-3}=6, s_{-2}=5, s_{-1}=2, s_{0}=7$. (Figure 5.4, shows behavior of solution of 5.4)

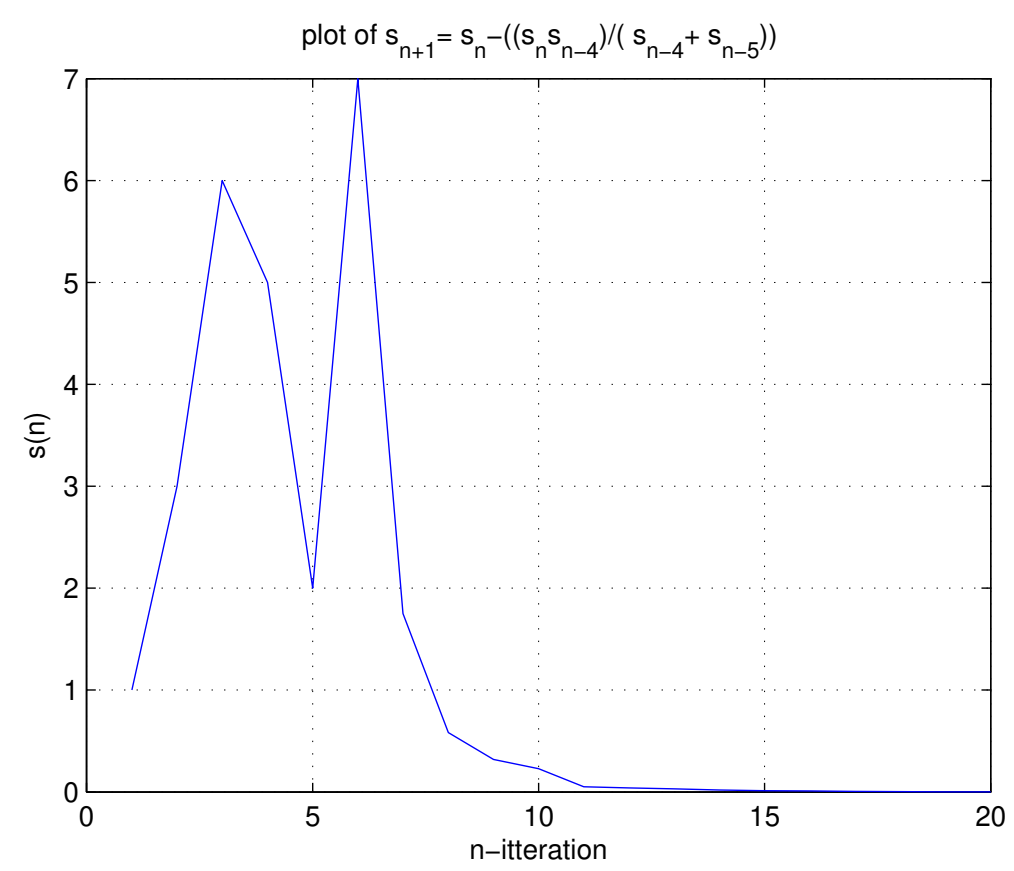

Figure 5.4: Shows behavior of Solution of 5.4

\subsection{Fourth equation}

In this section we deal with the specific form of the 1.1

$$
s_{n+1}=s_{n}-\frac{s_{n} s_{n-4}}{s_{n-4}-s_{n-5}}, \quad n=0,1, \ldots
$$

where the initial conditions $s_{-5}, s_{-4}, s_{-3}, s_{-2}, s_{-1}, s_{0}$ are arbitrary non-zero real numbers with $s_{-5} \neq s_{-4} \neq s_{-3} \neq s_{-2} \neq s_{-1} \neq s_{0}$.

Theorem 5.7. Let $\left\{s_{n}\right\}_{n=-5}^{\infty}$ be a solution of 5.2. Then every solution of it is periodic with period 24 . Moreover, $\left\{s_{n}\right\}_{n=-5}^{\infty}$ takes the form

$t, q, p, r, k, l, \frac{t l}{l-k}, \frac{t q l}{(t-q)(q-p)}, \frac{t q p l}{(t-q)(q-p)(p-r)}, \frac{t q p r l}{(t-q)(q-p)(p-r)(r-k)}, \frac{t q p r k l}{(t-q)(q-p)(p-r)(r-k)(k-l)}, \frac{-t p r k l}{(q-p)(p-r)(r-k)(k-l)}$, 
or,

$$
\begin{aligned}
& s_{24 n-5}=t, \\
& s_{24 n-3}=p, \\
& s_{24 n-1}=k \text {, } \\
& s_{24 n+1}=\frac{t l}{l-k}, \\
& s_{24 n+3}=\frac{t q p l}{(t-q)(q-p)(p-r)}, \\
& s_{24 n+5}=\frac{\text { tqprkl }}{(t-q)(q-p)(p-r)(r-k)(k-l)}, \\
& s_{24 n+7}=\frac{t r k l}{(p-r)(r-k)(k-l)}, \\
& s_{24 n+9}=\frac{t l}{(k-l)}, \\
& s_{24 n+11}=-q \text {, } \\
& s_{24 n+13}=-r \text {, } \\
& s_{24 n+15}=-l \text {, } \\
& s_{24 n+17}=\frac{-t q l}{(t-q)(q-p)}, \\
& s_{24 n+19}=\frac{-t q p r l}{(t-q)(q-p)(p-r)(r-k)}, \\
& s_{24 n+21}=\frac{t p r k l}{(q-p)(p-r)(r-k)(k-l)}, \\
& s_{24 n+23}=\frac{t k l}{(r-k)(k-l)},
\end{aligned}
$$

$$
\begin{aligned}
& s_{24 n-4}=q, \\
& s_{24 n-2}=r \text {, } \\
& s_{24 n}=l \text {, } \\
& s_{24 n+2}=\frac{t q l}{(t-q)(q-p)}, \\
& s_{24 n+4}=\frac{t q p r l}{(t-q)(q-p)(p-r)(r-k)}, \\
& s_{24 n+6}=\frac{-t p r k l}{(q-p)(p-r)(r-k)(k-l)}, \\
& s_{24 n+8}=\frac{-t k l}{(r-k)(k-l)}, \\
& s_{24 n+10}=-t, \\
& s_{24 n+12}=-p, \\
& s_{24 n+14}=-k, \\
& s_{24 n+16}=\frac{-t l}{(t-q)}, \\
& s_{24 n+18}=\frac{-t q p l}{(t-q)(q-p)(p-r)}, \\
& s_{24 n+20}=\frac{- \text { tqprkl }}{(t-q)(q-p)(p-r)(r-k)(k-l)}, \\
& s_{24 n+22}=\frac{-t r k l}{(p-r)(r-k)(k-l)}, \\
& s_{24 n+24}=\frac{-t l}{(k-l)} .
\end{aligned}
$$

where $s_{-5}=t, s_{-4}=q, s_{-3}=p, s_{-2}=r, s_{-1}=k, s_{0}=l$

Proof. The proof is left to the reader.

Example 5.8. Assume $s_{-5}=2, s_{-4}=17, s_{-3}=15, s_{-2}=14, s_{-1}=19, s_{0}=11$. (See Figure 5.5 for the periodic behavior of 5.5) and $s_{-5}=-2, s_{-4}=17, s_{-3}=15, s_{-2}=-8, s_{-1}=19, s_{0}=1$. (See Figure 5.6)

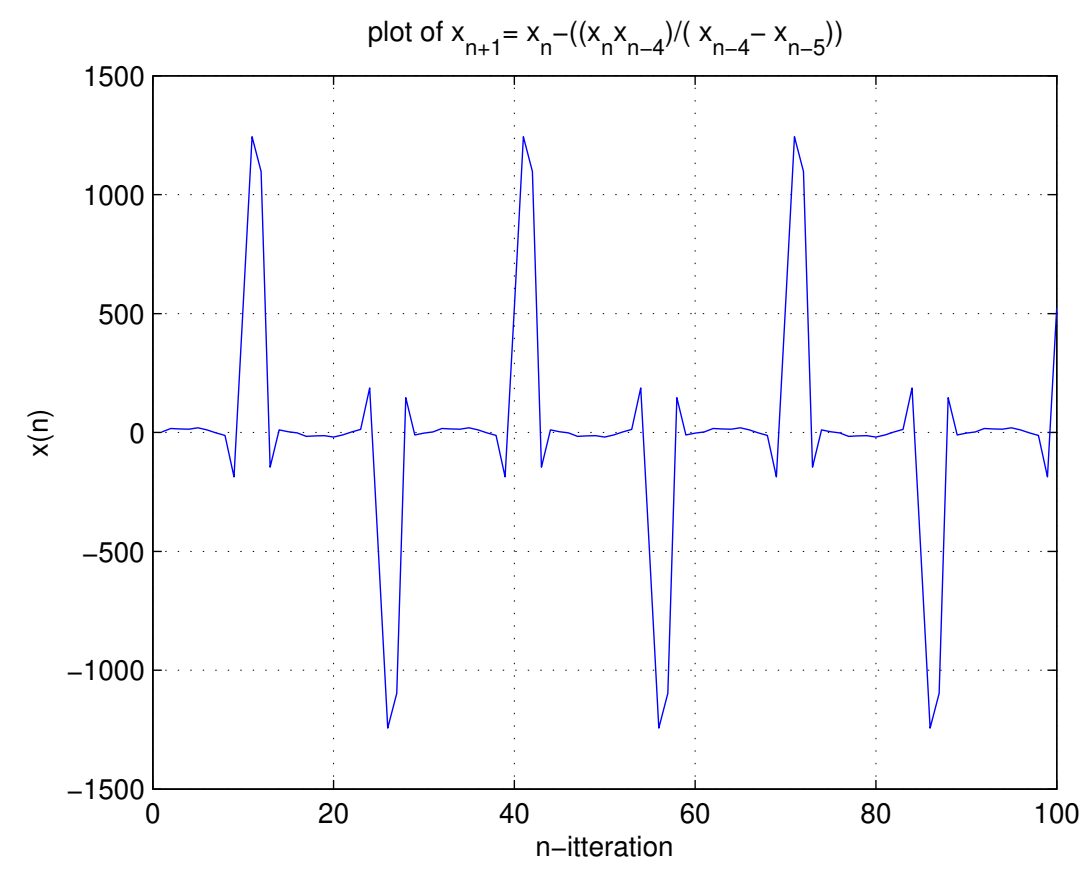

Figure 5.5: Shows periodic solution of 5.5 


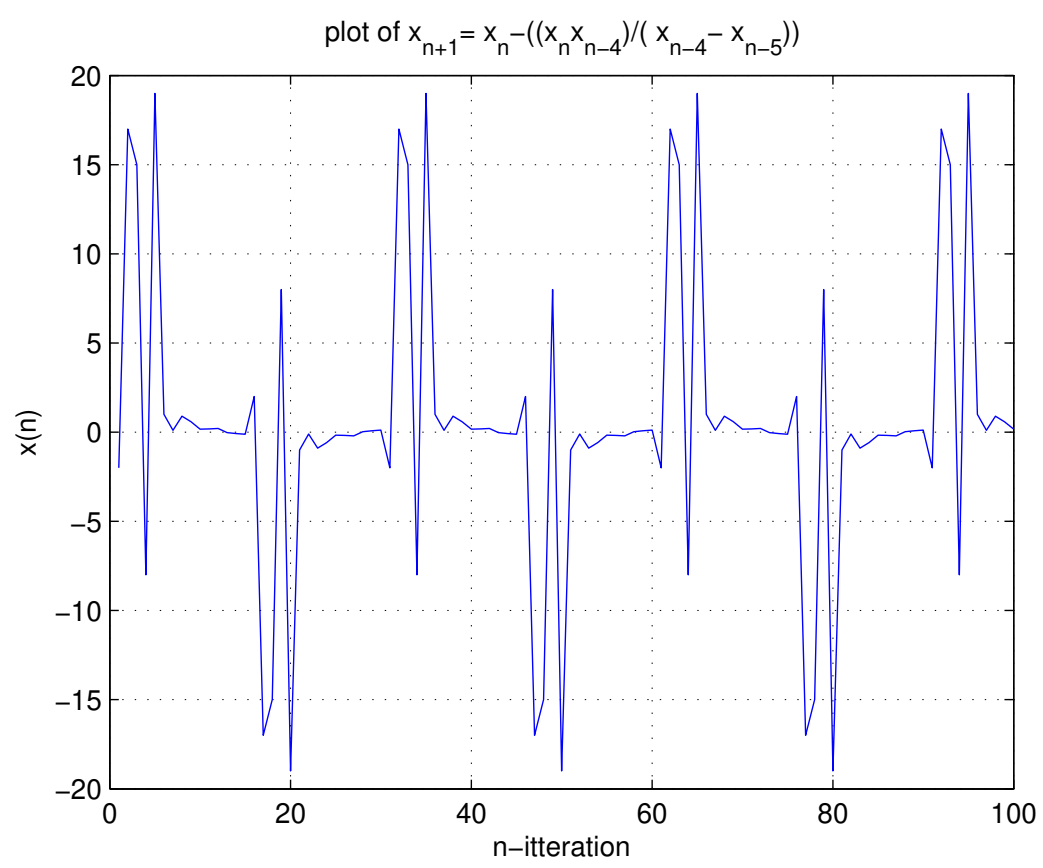

Figure 5.6: Shows periodic solution of 5.5

\section{Conclusion}

In This paper we studied global stability, boundedness and the solutions of some special cases of equation 1.1. In Section 3 we proved when $\beta(\gamma+3 \delta)<(\gamma+\delta)^{2}(1-\alpha), 1.1$ has local stability. We proved in the same section that the unique equilibrium of equation 1.1 is globally asymptotically stable if $\gamma(1-\alpha) \neq \beta$. In Section 4 we showed that the solution of equation 1.1 is bounded if $\left(\alpha+\frac{\beta}{\gamma}\right)<1$. In Section 5 , we obtained the expression and closed form solution of four special cases of equation 1.1 and gave numerical examples of each of the case, with different initial values.

\section{References}

[1] R.E. Mickens, Difference Equations, Van Nostrand Reinhold Comp, New York, 1987.

[2] M.C. Mackey, L. Glass, Oscillation and chaos in physiological control system, Science, 197(1977), 287-289.

[3] M.R.S. Kulenovic, G. Ladas, Dynamics of Second Order Rational Difference Equations with Open Problems and Conjectures, Chapman \& Hall / CRC Press, 2001.

[4] R.J. Beverton, S.J. Holt, On the Dynamics of Exploited Fish Populations, Fish Invest., London, 1957.

[5] R. DeVault, G. Dial, V.L., Kocic, G. Ladas, Global behavior of solutions of $x_{n+1}=a x_{n}+f\left(x_{n}, x_{n-1}\right)$, Journal of Difference Equations and Applications, 3(3-4)(1997), 311-330.

[6] E.M. Elsayed, Qualitative behaviour of difference equation of order two, Mathematical and Computer Modelling, 50(2009),1130-1141.

[7] Elabbasy, E. M. Elsayed, On the difference equation, $x_{n+1}=\frac{. \alpha x_{n-l}+\beta x_{n-k}}{A x_{n-l}+B x_{n-k}}$, Acta Mathematica Vietnamica, 33(2008), 85-94.

[8] R. Karatas, C. Cinar, D. Simsek, On positive solutions of the difference equation $x_{n+1}=\frac{x_{n-5}}{1+x_{n-2} x_{n-5}}$, Int. J. Contemp. Math. Sci., 1(10)(2006), 495-500.

[9] H. El-Metwally, E. M. Elsayed, Dynamics of a rational difference equation $x_{n+1}=\frac{a x_{n-l} x_{n-k}}{b x_{n-p}+c x_{n-q}}$, Chinese Annals of Mathematics series B, 15(5)(2013), $852-857$.

[10] E. M. Elsayed, Behavior and expression of the solutions of some rational difference equations, Journal of Computational Analysis and Applications, 15(1)(2013), 73-81.

[11] M. Saleh, M. Aloqeili, On the difference equation $y_{n+1}=A+\frac{y_{n}}{y_{n-k}}$, Appl. Math. Comput. 176(1)(2006), 359-363.

[12] E. M. Elsayed, A. Khaliq, Global attractivity and periodicity behavior of a recursive sequence, J. Comput. Anal. Appl., 22(2)(2017), 369-379.

[13] C. Cinar, On the positive solutions of the difference equation $x_{n+1}=\frac{a x_{n-1}}{1+b x_{n} x_{n-1}}$, Appl. Math. Comp., 156(2004) 587-590.

[14] S. E. Das, M.Bayram, On a System of Rational Difference Equations, World Applied Sciences Journal, 10(11)(2010), 1306-1312.

[15] Q. Din, E. M. Elsayed, Stability analysis of a discrete ecological model, Computational Ecology and Software, 4(2)(2014), 89-103.

[16] E. M. Elabbasy, H. El-Metwally, E. M. Elsayed, On the difference equations $x_{n+1}=\frac{\alpha x_{n-k}}{\beta+\gamma \prod_{i=0}^{k} x_{n-i}}$, J. Conc. Appl. Math., 5(2) (2007), 101-113.

[17] E. M. Elabbasy, H. El-Metwally, E. M. Elsayed, Qualitative behavior of higher order difference equation, Soochow Journal of Mathematics, 33(4)(2007), 861-873.

[18] E. M. Elabbasy, H. El-Metwally, E. M. Elsayed, On the Difference Equation $x_{n+1}=\frac{a_{0} x_{n}+a_{1} x_{n-1}+\ldots+a_{k} x_{n-k}}{b_{0} x_{n}+b_{1} x_{n-1}+\ldots+b_{k} x_{n-k}}$, Mathematica Bohemica, 133(2) (2008), 133-147.

[19] R. Agarwal, Difference equations and inequalities, Theory, methods and applications, Marcel Dekker Inc., New York, 1992.

[20] H. Chen, H. Wang, Global attractivity of the difference equation $x_{n+1}=\frac{x_{n}+\alpha x_{n-1}}{\beta+x_{n}}$, Appl. Math. Comp., 181(2006),1431-1438.

[21] E. M. Elsayed, Dynamics of a Recursive Sequence of Higher Order, Communications on Applied Nonlinear Analysis, 16(2)(2009), 37-50.

[22] A. Khaliq, E. M. Elsayed, The Dynamics and Solution of some Difference Equations, Journal of Nonlinear Sciences and Applications, 9(3) (2016), $1052-1063$

[23] A. Khaliq, On the Solution and Periodic Nature of Higher-order Difference Equation, Mathematical Sciences Letters, 6(2)(2017), 177-186.

[24] A. Khaliq, E. M. Elsayed, Qualitative properties of difference equation of order six, Mathematics, 4(2016), 24. 
[25] V. L. Kocic, G. Ladas, Global Behavior of Nonlinear Difference Equations of Higher Order with Applications, Kluwer Academic Publishers, Dordrecht,

[26] A. S. Kurbanli, On the Behavior of Solutions of the System of Rational Difference Equations, World Applied Sciences Journal, 10(11) (2010), 1344-1350.

[27] R. Memarbashi, Sufficient conditions for the exponential stability of nonautonomous difference equations, Appl. Math. Letter, 21 (2008), $232-235$.

[28] A. Neyrameh, H. Neyrameh, M. Ebrahimi, A. Roozi, Analytic solution diffusivity equation in rational form, World Applied Sciences Journal, 10(7)(2010), 764-768. 